Юань Тао ${ }^{1}$, В. П. Захаров

КОРПУСНО-ОРИЕНТИРОВАННЫЙ АНАЛИЗ УНИВЕРСАЛИИ «ИНОСТРАНИЗАЦИЯ" В КОНСТРУКЦИЯХ С ПРЕДЛОГОМ «对» (DUİ) В НАУЧНЫХ ТЕКСТАХ, ПЕРЕВЕДЕННЫХ С РУССКОГО ЯЗЫКА НА КИТАЙСКИЙ ${ }^{1}$

${ }^{1}$ Шэньсийский педагогический университет, Китайская Народная Республика, 710062, Xi'an, Chang'an Road (South), 199

2 Санкт-Петербургский государственный университет, Российская Федерация, 199034, Санкт-Петербург, Университетская наб., 7-9

Предложения с предлогом «对» (dui) в переводоведении, как правило, изучались в рамках «европеизации» китайского языка или, более конкретно, в рамках отношений между английским и китайским языками. В статье предпринята попытка изучить эти конструкции в рамках русско-китайского перевода. При изучении универсалии перевода «иностранизация» для данного типа предложений переводной язык рассматривается в двух аспектах: длина конструкций с предлогом «对» и грамматические функции. Одной из особенностей предложений c 对 в переводном языке является увеличение размера конструкций. Наблюдается также иностранизация грамматических функций. Особенности предложений с 对, названные выше, во многом определяются степенью учета особенностей русского языка переводным китайским. Библиогр. 27 назв. Табл. 3.

Ключевые слова: предложения с 对, длина конструкции, грамматическая иностранизация, универсалия «иностранизация».

\title{
ON THE FEATURES OF DUI CONSTRUCTION PATTERNS IN TRANSLATIONAL CHINESE FROM RUSSIAN ON THE BASIS OF PARALLEL CORPUS
}

Yuan Tao ${ }^{1}$, Victor P.Zakharov ${ }^{2}$

1 Shaanxi Normal University, 710062, China; Xiłan, Chang'an Road (South), 199

2 Saint Petersburg State University, 7-9, Universitetskaya nab., St. Petersburg, 199034, Russian Federation

Research on Dui constructions has been mostly conducted among English-Chinese translation in the context of Europeanization. With the help of a self-built parallel corpus, this article has attempted to explore its construction pattern features in translational Chinese from Russian in terms of its syntactic structure and grammatical functions. This research focuses its attention on the following hypotheses: 1) the construction's load capacity has been found to increase a great deal by comparing the numbers of closed, embedded Dui constructions in both translational corpus and a compatable corpus, 2) foreignization in grammatical functions has been confirmed by comparing the numbers of Dui constructions as subjects, objects, those as adverbials, and modifiers in both corpora. The article concludes that, although features of Dui construction patterns have been affected by many factors, the common underlying ones are: features of translational choice, genre factors, fusion or sublation of Russian in the translational Chinese. Translators are prone to follow the convention of source language in the course of their translation of academic texts. Compared with the translated version of literary texts, the translated versions of academic texts are obviously characteristic of original texts in terms of their content and structure. Refs. 27. Tables 3.

Keywords: Dui sentence patterns, construction's length, grammatical foreignization.

1 Исследование поддержано грантом Бюро Национального фонда социальных и гуманитарных наук Китайской Народной Республики № 13ВYY026 «Исследование перевода тематических текстов на основе параллельного корпуса русского и китайского языков».

(C) Санкт-Петербургский государственный университет, 2017 


\section{1. Введение}

В настоящем исследовании ставится задача изучить вопрос об особенностях конструкций с предлогом «对» в текстах, переведенных с русского языка на китайский. Предлог «对» (dui) - это предлог направления действия, он вводит дополнение адресата, на русский язык переводится беспредложным дательным падежом или разными предлогами («по отношению к», «относительно», «о» и др.) с соответствующей конструкцией.

При переводе с одного языка на другой наблюдаются некоторые закономерности, которые в переводоведении получили название универсалий [1]. Эти универсалии показывают характер и степень влияния языка исходного текста на язык перевода.

Статья посвящена универсалии «иностранизация» (англ. “foreignization”), означающей отдаление языка, представленного текстом перевода (будем называть его «переводной язык»), от оригинального языка, которым пользуются его носители (будем называть его «целевой язык»).

В работах китайских ученых предложения с 对 признаются типичной структурой китайского языка, где предлог «对» является словом-узлом конструкции. Отмечается, что в использовании предлога «对» и подобного ему предлога «对于» в переводном языке проявляется явление европеизации [2, с. 18].

Европеизация в целом - это принятие идей и ценностей европейской культуры как истинных, прогрессивных, этически оправданных и совершенных [3]. И перевод является одним из «двигателей» этой европеизации.

Мы постараемся разобраться, насколько обоснована идея иностранизации предложений с 对. Этот вопрос вновь приобретает свою актуальность в связи с возникшим в последние годы интересом к европеизации китайского языка [4-7], к отношениям между переводом и европеизацией [8].

Универсалии (“translation universals”) - это языковые особенности именно переводного текста. Универсалии перевода подробно изучены и описаны М.Бейкер $[1 ; 9]$. Переводной язык выступает в качестве посредника между двумя языками $[10$, c. 43$]$. Данная точка зрения имеет свою историю. Она получила развитие начиная с понятий “inter-language" [11], "third code" [12] через "hybrid language" [13] и заканчивая понятием "features of translation” [14].

До сих пор в основном вместо понятия «иностранизация» ученые пользовались терминами «европеизация» и «проникновение языка оригинала». Проникновение языка оригинала обычно анализируется в аспекте контактов двух языков. Вслед за работами П.Ньюмарка (P. Newmark) и Дж. Тури (J. Toury) проникновение языка оригинала стало исследоваться и в аспекте переводоведения. «В переводе везде имеет место вмешательство исходного языка, это характерное явление перевода» [15, с. 78]. «Перевод - это прежде всего подражание. Влияние оригинала выражается в том, что особенности исходного языка проникают в переводной текст» $[15$, с. 80]. Термин «проникновение языка оригинала» также связывают с «интеръязыком» $[16$, с. 13] и проникновением английского языка в китайский $[17 ; 18]$. Таким образом, явление и понятие «проникновение языка оригинала» тесно связано с переводной иностранизацией. Нас прежде всего интересует проявление иностранизации в тексте, как это когда-то очень давно постулировал Ф. Шляйермахер [19]. 
Иностранизацию можно считать антонимом термина «доместикация» (также «нормализация»). Доместикация и иностранизация обычно рассматриваются как два стиля перевода, когда переводчик «оглядывается» на домашнего читателя (доместикация) или, наоборот, на исходный текст (иностранизация).

\section{2. Проект исследования}

2.1. Постановка задачи. Наше исследование конструкций с 对 основано на сравнительном анализе статистических данных, полученных из текстов параллельного корпуса (корпус исходных и переводных текстов) с соответствующими данными сопоставимого корпуса (comparable corpus), представляющего собой подкорпус оригинальных китайских текстов.

2.2. Предмет исследования. Конструкции с предлогом «对» образуют «смысловую синтагму». Каждая отдельная смысловая синтагма имеет главное слово. В качестве таких главных слов допускается использование статических слов, элементов предикативного характера или минор-предложений ${ }^{2}$. Предложения с 对 формируют относительно закрытые конструкции. Данные конструкции не могут расширяться влево, допускается лишь постановка небольшого числа слов справа от 对, между 对 и главным словом.

2.3. Корпус. Базу исследования составили параллельный корпус русского и китайского языков и сопоставимый корпус китайского языка (научные тексты гуманитарной и социальной тематики). Параллельный корпус включает 14 монографий из области политики, международных отношений, лингвистики, литературоведения и переводоведения на русском языке и их переводы на китайский. В этих монографиях представлены тексты современного русского научного языка, переведенные за последние 30 лет. Сопоставимый корпус включает 10 научных монографий на китайском языке из тех же предметных областей, изданных за последние 16 лет. Совпадение тематики и стиля текстов параллельного и сопоставимого корпусов обеспечивает достоверность результатов сопоставления особенностей переводного и оригинального китайского языка [20].

Объем корпуса составляет 10000000 токенов. Для исследования были выбраны три подкорпуса (см. табл. 1).

Таблица 1. Характеристика корпусов

\begin{tabular}{|l|c|c|c|}
\hline \multirow{2}{*}{} & \multicolumn{2}{|c|}{$\begin{array}{c}\text { Параллельный корпус } \\
\text { (объем в токенах) }\end{array}$} & $\begin{array}{c}\text { Сопоставимый корпус } \\
\text { (объем в токенах) }\end{array}$ \\
\cline { 2 - 4 } & Русский язык & Китайский язык & Китайский язык \\
\hline $\begin{array}{l}\text { Политика и международные } \\
\text { отношения }\end{array}$ & 418100 & 710856 & 657718 \\
\hline Лингвистика & 568738 & 855326 & 795546 \\
\hline Литература & 208643 & 315258 & 316328 \\
\hline Итого & 1193481 & 1881440 & 1769592 \\
\hline
\end{tabular}

${ }^{2}$ Минор-предложения - особое понятие китайской грамматики, они соответствуют какойлибо части сложного предложения. 
Как видно из таблицы 1, количество токенов китайского языка в параллельном корпусе и сопоставимом корпусе практически одинаково.

\section{3. Экспериментальное исследование}

3.1. Длина конструкций с 对. Длина конструкции - это количество лексических единиц в ее составе. В оборотах с 对 под длиной конструкции подразумевается количество лексем, которые входят в текстовый интервал от 对 (или 对于) до главного слова, стоящего справа от 对 или 对于, включая само главное слово. В нашем исследовании под лексемой понимается токен (иероглиф), таким образом, длина конструкции - это количество токенов в интервале от 对 (или 对于) до главного слова.

Анализ показал, что в корпусе переводных текстов насчитывается 614 конструкций с 对, в сопоставимом корпусе их всего 485. Информация о количестве конструкций с разной длиной в двух корпусах приведена в таблице 2.

Таблица 2. Распределение конструкций с 对 в двух корпусах по длинам

\begin{tabular}{|c|c|c|c|c|c|c|c|c|c|c|c|}
\hline & \multicolumn{9}{|c|}{ Длина конструкции в токенах } & \multirow{3}{*}{$\begin{array}{c}\text { Общее } \\
\text { количество } \\
\text { конструкций } \\
\text { с 对 }\end{array}$} & \multirow{3}{*}{$\begin{array}{c}\text { Средняя } \\
\text { длина } \\
\text { конструкции }\end{array}$} \\
\hline & 1 & 2 & 3 & 4 & 5 & 6 & 7 & 8 & 9 & & \\
\hline & \multicolumn{9}{|c|}{ Количество конструкций данной длины } & & \\
\hline $\begin{array}{l}\text { Корпус переводных } \\
\text { текстов }\end{array}$ & 51 & 44 & 56 & 98 & 138 & 125 & 73 & 26 & 3 & 614 & 4,70 \\
\hline $\begin{array}{l}\text { Сопоставимый } \\
\text { корпус }\end{array}$ & 53 & 47 & 99 & 109 & 73 & 68 & 26 & 10 & 0 & 485 & 3,95 \\
\hline
\end{tabular}

Средняя длина конструкции получается делением суммы длин всех конструкций с 对 в данном корпусе на количество конструкций. Так, для корпуса переводных текстов она вычисляется следующим образом: $\left(1^{\star} 51+2^{\star} 44+3^{\star} 56+4^{\star} 98+\right.$ $\left.5^{\star} 138+6^{\star} 125+7^{\star} 73+8^{\star} 26+9^{\star} 3\right): 614=4,70$.

Мы видим, что средняя длина конструкций с 对 в корпусе переводных текстов превосходит среднюю длину конструкций в сопоставимом корпусе. В нем чаще всего встречаются конструкции с длиной 4-6, в то время как в текстах сопоставимого корпуса оптимальная длина 3-4.

Для русского языка характерны постпозитивные определения с использованием ряда слов в родительном падеже справа. Однако в китайском языке определения могут находиться лишь в препозиции, при этом отсутствует такой показатель связи между определением и главным словом, как падеж. Логическую цепочку определений в китайском языке можно построить лишь при помощи порядка слов. В этом случае увеличение длины конструкции непременно ведет к понижению читабельности.

Итак, для конструкций с 对 в целевом китайском характерна закрытость. Подвергаясь влиянию открытых конструкций русского языка, длина конструкций с 对 в переводном языке имеет тенденцию к увеличению. Таким образом, в переводном 
тексте проявляются особенности, характерные для исходного языка, т.е. наблюдается явление иностранизации.

3.2. Синтаксическая иностранизация. В рассматриваемых нами конструкциях c对 наблюдается также синтаксическая иностранизация. «Переводчик, перенося синтаксические особенности исходного текста в рамки целевого языка, изменяет формы целевого языка, что представляет собой синтаксическую иностранизацию» $[21$, с. 369].

В таблице 3 приводятся сведения об использовании конструкций с 对 в качестве различных членов предложений в двух корпусах. Напомним, что общее количество конструкций с 对 в корпусе переводных текстов составляет 614, а в сопоставимом корпусе - 485 .

Таблица 3. Сравнение частоты синтаксических функций конструкций с 对

\begin{tabular}{|c|c|c|c|c|c|c|c|c|}
\hline Синтаксическая функция & 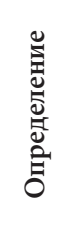 & 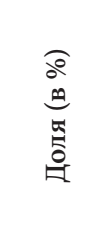 & 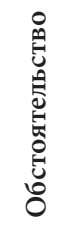 & 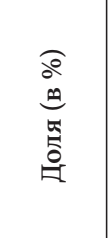 & 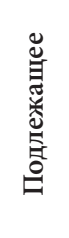 & 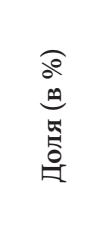 & : & 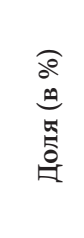 \\
\hline Корпус переводных текстов & 144 & $23,5 \%$ & 212 & $34,5 \%$ & 225 & $36,6 \%$ & 33 & $5,4 \%$ \\
\hline Сопоставимый корпус & 216 & $44,5 \%$ & 226 & $46,6 \%$ & 38 & $7,8 \%$ & 5 & $1,0 \%$ \\
\hline
\end{tabular}

Данные таблицы 3 свидетельствуют о том, что синтаксические роли конструкций с 对 в предложениях двух корпусов отличаются. Основные синтаксические функции конструкций с 对 в целевом китайском языке - определение $(44,5 \%)$ и обстоятельство (46, 6 \%). В переводном китайском выше всего процент конструкций с 对 в функции подлежащего (36, 6 \%). Также конструкции с 对 в переводном китайском чаще выполняют функцию дополнения, чем в целевом (5, 4 \% против $1 \%)$. Доля конструкций с 对 в функции обстоятельства в переводном корпусе больше доли конструкций с 对 в функции определения примерно на $10 \%$, в то время как в целевом языке они практически равны.

В языке перевода часто присутствует предлог «对», соответствующий русским предлогам «для», «К», «по», «при», при этом конструкции с ним, уподобляясь конструкциям русского языка, сохраняют функцию обстоятельства, в то время как предложные обороты китайского языка обычно выступают в качестве определений.

Яркое проявление иностранизации синтаксических функций оборотов с 对 это выполнение ими роли подлежащего и дополнения. Характерной особенностью научного стиля русского языка является частое использование отглагольных существительных, которые соответствуют глаголам по семантике и структурной валентности и в то же время имеют формы и синтаксические функции существительного. В предложении они могут выступать в качестве подлежащего и дополнения. Конструкции с 对, являющиеся переводом русских отглагольных существительных, сохраняют синтаксические функции исходного языка - аналогично русскому они выступают в роли подлежащего или дополнения. 


\section{4. Заключение}

Проведенное исследование явным образом показало наличие иностранизации в предложениях с 对 в переводном языке. Обороты с 对, являющиеся переводом с русского языка, представляют собой относительно открытые структуры, длина которых больше длины конструкций оригинального китайского языка.

Синтаксические функции оборотов с 对 в качестве элементов предложения в переводном и целевом языках также отличаются. Очевидно, что отглагольные существительные русского языка и обороты с 对 имеют явную взаимосвязь. При переводе форма и лексика текста-оригинала в большой степени ограничивают образ мышления и выбор переводчика, что, в свою очередь, «заставляет» перевод по содержанию и форме соответствовать оригиналу.

Можно утверждать, что в процессе перевода или в процессе контакта двух языков, факторы, влияющие на выбор переводчика, выстраиваются следующим образом: структура языка оригинала - контекст языка оригинала - структура языка перевода - контекст языка перевода - фактор психологии переводчика [22, c. 233]. Первое, на что обращает внимание переводчик, - это структура, грамматика и словарный состав исходного языка. Поэтому эффект проникновения языка оригинала - распространенное явление, характерное для большинства переводов.

Принцип перевода научных текстов таков, что язык перевода стремится к единству с языком оригинала. «Соответствие реальному образу текста оригинала» - базовое правило академического перевода [23]. «Принцип верности» - важное правило, которому необходимо следовать при переводе классических работ [24]. В отличие от перевода литературных произведений, при переводе научного текста переводчик в большей степени стремится сохранить структуру и содержание текста оригинала.

При рассмотрении данного вопроса с позиции европеизации необходимо отметить, что после Движения 4-го мая³ наряду с англоязычными литературными произведениями в Китай в большом количестве стали поступать произведения и на других индоевропейских языках, в том числе произведения русской литературы и труды по общественным наукам на русском языке. Современные научные тексты, переведенные на китайский язык, являются переводами не только с английского языка, но и с русского, французского и других языков, морфологическая изменчивость которых разнообразнее, а синтаксические функции специфичнее. Поэтому нельзя оставить без внимания влияние русского и других индоевропейских языков на китайский язык и степень восприятия этих языков китайским языком.

Перспективу нашего исследования может составить изучение проблемы на более широком языковом материале за счет привлечения к анализу других закрытых конструкций переводного китайского языка.

Изучаемая в статье иностранизация относится к категории «многогранной европеизированной грамматики» [25, с.84]. Хотя функции оборотов с 对 в роли

${ }^{3}$ Движение 4-го мая - это массовое антиимпериалистическое (преимущественно антияпонское) движение в Китае, возникшее в мае-июне 1919 г. под влиянием Октябрьской революции в России. В широком смысле Движение 4-го мая обозначило поворот во взглядах китайской интеллигенции: массовую переориентацию с традиционной культуры на вестернизацию. Движение затронуло все стороны интеллектуальной жизни Китая. 
подлежащего и дополнения не являются чем-то запрещенным в китайском языке, но их заметное присутствие в текстах на китайском языке стало проявляться только под влиянием индоевропейских языков [25]. Причем в переводных текстах функция подлежащего в оборотах с 对, как мы показали, становится преобладающей. Естественно, проявление универсалии иностранизации относится не только к изучаемым здесь конструкциям и не только к переводному языку. Переводной язык является частью китайской языковой культуры и влияет на язык в целом. И истоки европеизации китайского языка, по мнению ряда ученых, кроются именно в переводе [26, с. 501; 27, с. 32].

Имеется немало работ, показывающих, как менялся китайский язык на протяжении последнего века. Но для того чтобы показать это доказательно и количественно, требуется создание репрезентативного диахронического корпуса. И хотя у нас такого корпуса пока нет, мы рассчитывает изучить явление иностранизации в области лексического состава китайского языка на основе корпуса Google books Ngram Viewer, содержащего в своем составе диахронический подкорпус китайского языка.

\section{Литература}

1. Baker M. Corpus linguistics, translation studies: implications and applications // Text and Technology: In Honor of John Sinclair. Amsterdam; Philadelphia: John Benjamnis, 1993. P. 233-250.

2. Xie Yaoji. Hanyu yufa ouhua zongshu // Yuwen yanjiu. 2001. No 1. P. 17-22. (In Chinese).

3. Баженова Т.П., Семина В.С. Сущность европеизации русской культуры // Аналитика культурологии. 2008. № 10. С. 174-185.

4. Song Wenhui. Zailun hanyu mingcixing binglie jiegou de «ouhua» shuo // Yuyan jiaoxue yu yanjiu. 2016. No 2. P. 80-91. (In Chinese).

5. Wang Jian. Lun Dingweiliang yi «guanhua yuehan fuyinshu» yu hanyu ouhua baihuawen de lanshang // Waiyu yanjiu. 2015. No 6. P. 83-89. (In Chinese).

6. Huang Yi. «Ouhua» «difangse» yu «shijiexing» - lun «Wusi» xiangtu piping lilun de chuchaung // Lu Xun yanjiu yuekan. 2015. No 8. P.11-16. (In Chinese).

7. Жукаускене Т.С., Холдаенко И.С. Заимствования в китайском языке: влияние английского языка в условиях глобализации // Текст. Книга. Книгоиздание. 2015. № 1 (8). С.31-42.

8. Zhou Hongmin. Cong «ouhua wen» dao «fanyi ti» - fanyi yu hanyu yige shiji de «qing» yu «jie» // Yangzhou daxue xuebao. 2015. No. 4. P.117-123.

9. Baker M. A Corpus-based view of similarity, difference in translation // International Journal of Corpus Linguistics. 2004. Vol.9, No 2. P. 167-193.

10. Laviosa S. Corpus-based translation studies. Amsterdam; New York: Rodopi, 2002. 138 p.

11. Toury G. Interlanguage, its manifestations in translation // Meta. 1979. Vol.24, No 2. P. 223-231.

12. Frawley W. Prolegomenon to a theory of translation // Translation: Literary, Linguistic, Philosophical Perspectives. London: Associated University Press, 1984. P. 159-175.

13. Schäffner C., Adab B. The Idea of the hybrid text in translation: Contact as conflict across languages and cultures // Special Issue on Hybrid Texts and Translation. 2001. P. 167-181.

14. Olohan M. Introducing Corpora in Translation Studies. London; New York: Routledge, 2004. 236 p.

15. Newmark P. About Translation, Clevedon. Philadelphia. Adelaide: Multilingual Matters, 1991. 184 p.

16. Hopkinson C. Factors in linguistic interference: A case of study in translation // SKASE Journal of Translation and Interpretation. 2007. Vol.2, No 1. P.13-23.

17. Dai Guangrong. Yiwen yuanyu touguo xiaoying yanjiu // Shanghai, Shanghai Jiaotong daxue chubanshe. 2013. 223 p. (In Chinese).

18. Dai Guangrong, Xiao Zhonghua. Yiwen yuanyu touguo xiaoying yanjiu: jiyu hanyu fanyi yuliaoku // Fanyi jikan. 2011. Vol.62, No 4. P. 85-107. (In Chinese).

19. Schleiermacher F. On the different methods of translating // Theories of Translation: An Anthology of Essays from Dryden to Derrida / eds. R. Schulte, J. Biguenet. Chicago: University of Chicago Press, 1992. P. 67-69. (Original German version: Über die verschiedenen Methoden des Übersetzens. 1813). 
20. Тао Ю., Захаров В. П. Разработка и использование параллельного корпуса русского и китайского языков // Научно-техническая информация. Сер. 2. № 4. С. 18-29.

21. Qin Hongwu. Fanyi zhong de jufa yihua yu guihua // Waiyu jiaoxue yu yanjiu, 2000. No 5. P.368373. (In Chinese).

22. Tao Yuan. Shunyinglun shijiao xia de mohu yuyan fanyi yanjiu. Wuhan: Wuhan daxue chubanshe, 2014. 265 p. (In Chinese).

23. Zhang Yaquan. Lun wenxue fanyi d wenxian huiyi - yi Meierqing < qing chu Yangzhou wenhua> zhong yiben wei li // Nanjing daxue xuebao. 2005. No 3. P. 128-136. (In Chinese).

24. Zhu Daqiu. Guanyu jingdian zhuzuo fanyi de xuanxiang - yi $<$ Eluosi sixiang $>$ de yiwen wei li // Waiyu jiaoxue lilun yu shijian. 2010. No 3. P. 81-87. (In Chinese).

25. He Yang. Cong xiandai hanyu jieci zhong de ouhua xianxiang kan jianjie yuyan jiechu // Yuyan wenzi yingyong. 2004. No 4. P. 82-89. (In Chinese).

26. Wang Li. Zhongguo yufa lilun, <Wang Li wenji> (Diyi juan). Ji Nan: Shangdong jiaoyu chubanshe, 1984. 515 p. (In Chinese).

27. Xie Yaoji. Xiandai hanyu ouhua yufa gailun. Hong Kong: Guangming tushu gongsi, 1990. 309 p. (In Chinese).

Для цитирования: Юань Тао, Захаров В.П. Корпусно-ориентированный анализ универсалии «иностранизация» в конструкциях с предлогом «对» ('duì) в научных текстах, переведенных с русского языка на китайский // Вестник СПбГУ. Востоковедение и африканистика. 2017. Т. 9. Вып. 2. С.150-158. DOI: 10.21638/11701/spbu13.2017.203.

\section{References}

1. Baker M. Corpus linguistics, translation studies: implications and applications. In Baker M., Francis G. and Tognini-Bonelli E. (eds.). Text and Technology: In Honour of John Sinclair. Amsterdam \& Philadelphia, John Benjamins, 1993, pp.233-250.

2. Xie Yaoji. A literature review of Europeanization of Chinese Grammar. Study on Chinese, 2001, no. 1, pp. 17-22. (in Chinese)

3. Bazhenova T.P., Semina V.S. Sushchnost' evropeizatsii russkoi kul'tury [Substance of the europeanisation of the Russian culture]. Analitika kul'turologii [Culturology Analitics], 2008, no. 10, pp. 174185. (in Russian)

4. Song Wenhui. More on the Europeanization of Chinese NP Coordination Constructions. Language Teaching and Research, 2016, no. 2, pp. 80-91. (in Chinese)

5. Wang Jian. W. A. P. Martin's Translation of The Gospel of John in Mandarin Chinese and the Initiation of Europeanization of Vernacular Chinese. Languages Research, 2015, no. 6, pp. 83-89. (in Chinese)

6. Huang Yi. Local color and Universality of Europeanization: A study on critical theory initiation of dialect novel in May Fourth Movement. Monthly Review on Lu Xun, 2015, no. 8, pp. 11-16. (in Chinese)

7. Zhukauskene T.S., Holdaenko I. S. Zaimstvovaniya v kitaiskom yazyke: vliyanie angliiskogo yazyka v usloviyakh globalizatsii [Loanwords in Chinese: Global Influence of English]. Tekst. Kniga. Knigoizdanie [Text. Book. Publishing], 2015, no. 1(8), pp.31-42. (in Russian)

8. Zhou Hongmin. From Europeanized Chinese to Translationese: An Overview of a Century-long Controversy over the Impact of Translation on Chinese. Journal of Yangzhou University, 2015, no. 4, pp. 117123. (in Chinese)

9. Baker M. A. Corpus-based view of similarity, difference in translation. International Journal of Corpus Linguistics, 2004, vol. 9, no. 2, pp. 167-193.

10. Laviosa S. Corpus-based translation studies. Amsterdam \& NewYork, Rodopi, 2002.

11. Toury G. Interlanguage, its manifestations in translation. Meta, 1979, vol.24, no. 2, pp. 223- 231.

12. Frawley W. Prolegomenon to a theory of translation. Translation: Literary, Linguistic, Philosophical Perspectives. In Frawley W. (ed.). London, Associated University Press, 1984, pp. 159-175.

13. Schäffner C., Adab B. The Idea of the hybrid text in translation: Contact as conflict across languages and cultures. Special Issue on Hybrid Texts and Translation, 2001, pp. 167-181.

14. Olohan M. Introducing Corpora in Translation Studies. London, NewYork, Routledge, 2004. 236 p.

15. Newmark P. About Translation. Clevedon. Philadelphia. Adelaide, Multilingual Matters, 1991. 184 p.

16. Hopkinson C. Factors in linguistic interference: A case of study in translation. SKASE Journal of Translation and Interpretation, 2007, vol.2, no. 1, pp. 13-23.

17. Dai Guangrong. Source Language Shining Through in English-Chinese Translation. Shanghai, Shanghai Jiaotong University Press, 2013. 223 p. (in Chinese) 
18. Dai Guangrong, Xiao Zhonghua. Source Language Shining Through in Translational Language: A Corpus-based Study of Chinese Translation of English Passives. Translation Quarterly, 2011, vol.62, no.4, pp. 85-107. (in Chinese)

19. Schleiermacher F. On the different methods of translating. In Schulte R., Biguenet J. (eds). Theories of Translation: An Anthology of Essays from Dryden to Derrida. Chicago, University of Chicago Press, 1992, pp. 67-69. (Original German version: Über die verschiedenen Methoden des Übersetzens. 1813).

20. Tao Y., Zakharov V.P. The Development and Use of Russian-Chinese Parallel Corpus. Automatic Documentation and Mathematical Linguistics, no. 4. pp. 65-75.

21. Qin Hongwu. 2000. On syntactic foreignization and domestication in translation. Foreign Language Teaching Research, 2015, no. 5, pp. 368-373. (in Chinese)

22. Tao Yuan. A study on the translation of fuzzy language from the perspective of adaptation theory. Wuhan, Wuhan University Press., 2014. 265 p. (in Chinese)

23. Zhang Yaquan. On the translation of literature - a case study of Tobie Meyer-Fong's "Yangzhou culture" in the early Qing Dynasty. Journal of Nanjing University. 2005, no. 3, pp. 128 -136. (in Chinese)

24. Zhu Daqiu. A study on the translation of classic works - Taking "Russian spirit" translation as an example. Theory and practice of foreign language teaching, 2010, no. 3, pp. 81-87. (in Chinese).

25. He Yang. A Study of the Phenomenon of Europeanized Preposition in Modern Chinese. Applied Linguistics, 2004, no. 4, pp. 82-89. (in Chinese)

26. Wang Li. Chinese Grammatical Theory. Ji Nan, Shangdong Education Press, 1984. 515 p. (in Chinese)

27. Xie Yaoji. A brief introduction to Europeanization of Chinese Grammar. Hong Kong, Guangming Books Inc., 1990. 309 p. (in Chinese)

For citation: Yuan Tao, Zakharov V.P. On the Features of Dui Construction Patterns in Translational Chinese from Russian on the Basis of Parallel Corpus. Vestnik SPbSU. Asian and African Studies, 2017, vol.9, issue 2, pp. 150-158. DOI: 10.21638/11701/spbu13.2017.203.

Статья поступила в редакцию 14 октября 2016 г. Статья рекомендована в печать 28 февраля 2017 г.

Контактная информация

Юань Тао — доктор филологических наук; tao1973@mail.ru

Захаров Виктор Павлович - кандидат филологических наук; v.zakharov@spbu.ru

Yuan Tao - Dr. Sci Philology; tao1973@mail.ru

Zakharov Victor P. - PhD; v.zakharov@spbu.ru 